The purpose of this feature is to heighten awareness of specific adverse drug reactions (ADRs), discuss methods of prevention, and promote reporting of ADRs to the US Food and Drug Administration's (FDA's) MEDWATCH program (800-FDA-1088). If you have reported an interesting, preventable ADR to MEDWATCH, please consider sharing the account with our readers. Write to Dr. Mancano at ISMP, 200 Lakeside Drive, Suite 200, Horsham, PA 19044 (phone: 215-707-4936; e-mail: mmancano@temple.edu). Your report will be published anonymously unless otherwise requested. This feature is provided by the Institute for Safe Medication Practices (ISMP) in cooperation with the FDA's MeDWATCH program and Temple University School of Pharmacy. ISMP is an FDA MEDWATCH partner.

\section{BACTEREMIA IN PRETERM INFANTS RECEIVING PROBIOTICS}

The administration of probiotics to preterm babies has been shown to prevent necrotizing enterocolitis (NEC) and reduce all-cause mortality. The Swiss authors report 2 cases of bacteremia caused by the strain of bacteria present in the probiotic product Infloran administered to these preterm infants. Infloran contains Lactobacillus acidophilus $5 \times 10^{8}$ colony forming units (CFU) and Bifidobacterium infantis $5 \times 10^{8}$ CFU.

The first case occurred in a premature female infant weighing $867 \mathrm{~g}$. The infant was initiated on amoxicillin and gentamicin at birth; the antibiotics were discontinued after 3 days after a negative blood culture. The infant was started on enteral feedings of her mother's milk on the day of her birth. Infloran probiotic was administered to the infant enterally beginning on the fifth day of life (DOL) and continuing to the 14th DOL. On the infant's 14th DOL, she developed symptoms suggestive of sepsis with tachycardia, oxygen desaturation, and ileus. A complete blood count (CBC) at that time revealed leukocytosis $\left(28.5 \times 10^{9} / \mathrm{L}\right)$ with a left shift. An abdominal $\mathrm{x}$-ray revealed nonspecific findings with dilated bowel loops. Ceftazidime and vancomycin were initiated and continued for 7 days for suspected central linerelated sepsis. The infant developed a second episode of feeding intolerance and intestinal distention; on DOL 21, she was started on a 7-day course of imipenem.

On DOL 14, an anaerobic blood culture was obtained and was positive for B. infantis. The infant's clinical status began to improve over 2 weeks, but she then experienced a third episode of ileus. Testing revealed a small bowel obstruction. The infant

"Chair and Clinical Professor, Department of Pharmacy Practice, Temple University School of Pharmacy, Philadelphia, Pennsylvania; Clinical Advisor, Institute for Safe Medication Practices, Horsham, Pennsylvania 
required a laparotomy on DOL 40 that revealed ileoileal intussusception, which required resection of $15 \mathrm{~cm}$ of the ileum and primary anastomosis. The infant recovered postoperatively and was eventually discharged approximately 8 weeks later.

The second case occurred in another premature female infant weighing $1090 \mathrm{~g}$ whose initial clinical course was similar to the first patient. She received enteral feedings of her mother's milk on DOL 2 and was initiated on amoxicillin and gentamicin, which were eventually discontinued upon negative blood cultures on DOL 3. She received Infloran enterally from DOL 5 to DOL 9. On DOL 10, she experienced abdominal distention, tenderness, ileus, and rapid clinical deterioration with hypotension, lactic acidosis, thrombocytopenia, and coagulopathy requiring intubation and multiple transfusions with fresh frozen plasma, platelets, and blood. Her CBC revealed leukopenia $\left(2.0 \times 10^{9} / \mathrm{L}\right)$ with a left shift and an elevated C-reactive protein. The infant was diagnosed with NEC stage III and was treated with metronidazole, ceftazidime, and amikacin. Blood cultures taken on DOL 10 were positive for B. infantis.

Enteral feedings were restarted in the infant on DOL 22, but she experienced persistent feeding intolerance. On DOL 42, a laparotomy was performed that revealed intestinal necrosis and jejunal perforation requiring resection of $25 \mathrm{~cm}$ of small intestine and a primary anastomosis. The patient recovered postoperatively and was discharged approximately 6 weeks later.

The authors sequenced a total of $8 \mathrm{~B}$. infantis strains. They sequenced the $2 \mathrm{~B}$. infantis strains that had caused bacteremia in the infants as well as 6 strains of $B$. infantis that were obtained directly from capsules of Infloran. By utilizing comparative genomics, the authors were able to confirm that the strains isolated from each bacteremic infant originated from the probiotic product Infloran.

The authors point out that among greater than 5,000 newborns who were enrolled in clinical trials of probiotic prophylaxis of NEC, there were no cases of probiotic-associated sepsis reported (AlFelah $\mathrm{K}$, et al). However the authors were able to identify 2 additional case reports in the literature of bacteremia associated with probiotic use in premature newborns. The authors also make the point that while probiotic-associated bacteremia is likely to be a rare event, its true incidence could be underestimated. They reason that since anaerobic blood cultures are not always obtained in newborns with suspected infections, it is possible $B$. infantis infection could be overlooked. They recommend that future clinical trials include surveillance of blood cultures aimed at detecting anaerobic bacteria as well as longterm follow-up of infants with probiotic-associated bacteremia.

Bertelli C, Pillonel T, Torregrossa A, et al. Bifidobacterium longum bacteremia in preterm infants receiving probiotics. CID. 2015;60(March 15):924-927.

AlFaleh K, Anabrees J. Probiotics for prevention of necrotizing enterocolitis in preterm infants. Cochrane Database Syst Rev. 2014;4:CD005496.

\section{THROBBING HEADACHE ASSOCIATED WITH ENOXAPARIN USE}

A 60-year-old male was being treated for an upper left extremity thrombophlebitis with a therapeutic dose of enoxaparin $6000 \mathrm{IU} /$ day. The authors refer to the dosage as $6000 \mathrm{IU} /$ day, which would equate to $60 \mathrm{mg}$ of enoxaparin daily. This conversion is based on the Lovenox product labeling, which states that $10 \mathrm{mg}$ enoxaparin sodium has an approximate antifactor Xa activity of 1000 IU with reference to the World Health Organization's First International Low Molecular Weight Heparin Reference Standard. Approximately 1 hour after the patient received his first dose of enoxaparin, he experienced a throbbing headache. He described the pain as intense, localized in the occipital region, and accompanied by chest tightness. The patient reported that his headache spontaneously resolved 2 hours after onset of symptoms. Based on the severity and the temporal relationship of the patient's adverse reaction to enoxaparin, therapy was discontinued and an alternate treatment was initiated.

A medication history revealed that the patient did not take any concomitant drugs or herbal supplements, and his medical history revealed no personal or family history of migraine, cluster, or tension headaches. Laboratory parameters revealed normal hematologic tests, erythrocyte sedimentation rate, prostate-specific antigen, rheumatoid factor, thyroidstimulating hormone, antinuclear antibodies, creatinine, glomerular filtration rate (GFR), albumin, and liver function tests.

Ten days after the patient's initial reaction, his physician prescribed enoxaparin $6000 \mathrm{IU}$ (60 mg) daily again, and the patient experienced a throbbing headache with the same intensity. The patient's symptoms also occurred at 1 hour after enoxaparin administration and resolved upon drug withdrawal. On a third occasion, the patient was given a lower 
dosage of enoxaparin, $2000 \mathrm{IU}(20 \mathrm{mg})$ daily. Even with the enoxaparin dosage reduction, the patient's headache recurred, although it was less intense. At this time, his enoxaparin therapy was replaced with warfarin. The patient remained headache-free during 3 months of follow-up.

The authors evaluated this adverse event via the Naranjo scale; because the patient had a double positive rechallenge, the scale indicated a certain relationship between enoxaparin administration and the patient's throbbing headache. The authors then investigated the reports of heparin and low molecular weight heparins (LMWH) in 3 international databases: the US Food and Drug Administration's Adverse Event Reporting System (AERS), the Australian Database of Adverse Event Notifications (DAEN), and the Canadian Vigilance ADR Database. They found that the majority of headache reports were with heparin followed by enoxaparin. The authors surmised that enoxaparin most likely had the largest number of headache adverse drug reports, because of higher frequency of use versus other LMWH products.

The authors then investigated a possible mechanism of action by which heparin and LMWH could cause headache. They stated, "Heparin can downregulate the contraction pathway of vascular smooth cells; an effect involving various metabolic pathways such as the suppression of the production of endothelin, the stimulation of the release of nitric oxide (NO) and the increase of cyclic guanosine monophosphate (cGMP)." The authors also noted that increased NO synthesis has been shown to provoke headache. The authors warn that headache is likely a heparin class effect, and therefore clinicians should be aware of this potential adverse drug reaction in patients receiving heparin or LMWH.

Brusadelli T, Carnovale C, Gentili M, et al. Throbbing headache associated with enoxaparin administration: A case report, a review of the pharmacovigilance databases for similar cases and possible mechanisms [published online ahead of print March 2, 2015]. J Clin Pharm Ther. doi:10.1111/ jcpt.12257

Lovenox [product information]. Bridgewater, NJ: SanofiAventis; October 2013.

\section{DRESS REACTION FOLLOWING ISONIAZID TREATMENT}

A 71-year-old male presented to the hospital with complaints of a skin eruption. The patient had no history of medication allergies, but a medication history revealed that he had been receiving antituberculosis medications for the past 2 months to treat tuberculous meningitis. His daily medication regimen included isoniazid $300 \mathrm{mg}$, rifampin $450 \mathrm{mg}$, ethambutol $800 \mathrm{mg}$, and pyrazinamide $1000 \mathrm{mg}$. The patient's rash was described as a whole body generalized skin eruption. The patient's skin was exfoliating but not itching.

The patient's vital signs and the preponderance of his laboratory results were unremarkable. However, the following laboratory values were abnormal: neutrophils $46.8 \%$ (reference range, $54 \%-62 \%$ ), lymphocytes $21.9 \%$ (reference range, $25 \%-33 \%$ ), monocytes $20.9 \%$ (reference range, 3\%-7\%), eosinophils $9.8 \%$ (reference range, $1 \%-3 \%$ ), aspartate aminotransferase (AST) $90 \mathrm{IU} / \mathrm{L}$ (reference range, $<35 \mathrm{IU} / \mathrm{L}$ ), alanine aminotransferase (ALT) 74 IU/L (reference range, $<35$ IU/L), alkaline phosphatase 46 IU/L (reference range, 44-147 IU/L), lactate dehydrogenase (LDH) 607 IU/L (reference range, 100-190 IU/L), and total bilirubin $1.7 \mathrm{mg} / \mathrm{dL}$ (reference range, $0.3-1 \mathrm{mg} / \mathrm{dL}$ ).

The patient's antituberculin medications were all discontinued, and he was started on corticosteroid therapy. The patient's skin lesions gradually resolved over 3 weeks. However due to the concern over the possible suboptimal treatment of the patient's tuberculous meningitis, a medication reinitiation plan was undertaken. Rifampin was initiated at $150 \mathrm{mg}$ daily and increased to the patient's prior dosage of $450 \mathrm{mg}$ daily. At this time, the patient's skin lesions did not recur. However, when isoniazid was reinitiated at $100 \mathrm{mg}$ daily for 3 days, the patient's skin lesions on his whole body reappeared. The patient's isoniazid was stopped and he was diagnosed with a drug reaction with eosinophilia and systemic symptom (DRESS). The patient then received systemic methylprednisolone $2 \mathrm{mg} / \mathrm{kg}$ for 5 days, which was tapered over 2 weeks. The patient fully recovered from the effects of his isoniazid rechallenge. He then was managed with a second-line antituberculosis regimen of rifampin, streptomycin, and moxifloxacin. The patient has been followed for 3 months without any other complications.

The authors point out that rifampin is typically the most common offending drug associated with DRESS syndrome in patients receiving antituberculosis medications. This patient demonstrated that isoniazid was the possible cause of his DRESS syndrome, but the other drugs in his regimen were not rechallenged. So it is not known whether pyrazinamide or ethambutol were also causative agents of this patient's DRESS syndrome.

Lee JY, Seol YJ, Shin DW, et al. A case of the drug reaction with eosinophilia and systemic symptom (DRESS) following isoniazid treatment. Tuberc Respir Dis. 2015;78:27-30. 


\section{SIADH ASSOCIATED WITH GLIMEPIRIDE}

A 62-year-old Japanese male was noted to have a serum sodium of $125 \mathrm{mmol} / \mathrm{L}$ (reference range, 138-146 mmol/L), however his blood urea nitrogen (BUN), creatinine, adrenocorticotropic hormone (ACTH), cortisol, free triiodothyronine, and thyroxine levels were all within normal limits. In spite of the patient's hyponatremia, his plasma antidiuretic hormone $(\mathrm{ADH})$ was detectable at $0.8 \mathrm{pg} / \mathrm{mL}$ (reference range, $0.3-3.5 \mathrm{pg} / \mathrm{mL}$ ). His plasma osmolality decreased to $276 \mathrm{mOsm} / \mathrm{kg}$ and urinary osmolality and urinary sodium were $410 \mathrm{mOsm} / \mathrm{kg}$ and $134 \mathrm{mmol} / \mathrm{L}$, respectively.

A medication history revealed that the patient had been receiving the following medications: nicorandil (a potassium channel activator, vasodilator not available in the United States), atorvastatin, losartan, carvedilol, aspirin, isosorbide dinitrate, clopidogrel, and rabeprazole. Approximately 3 months earlier, the patient was initiated on the following medications for the treatment of his diabetes; voglibose $0.9 \mathrm{mg}$ daily (an $\alpha$-glucosidase inhibitor not available in the United States), alogliptin $25 \mathrm{mg}$ daily, metformin $2250 \mathrm{mg}$ daily, and glimepiride $0.5 \mathrm{mg}$ daily. At that time, his plasma glucose and $\mathrm{HbA}_{1 \mathrm{c}}$ were $140 \mathrm{mg} / \mathrm{dL}$ and $7.2 \%$, respectively, and his serum sodium was within normal limits $(138 \mathrm{mmol} / \mathrm{L})$. The patient's medication regimen was unchanged prior to his presentation with hyponatremia.

The authors state, "Based on the clinical, biochemical and radiological evaluations of the patient there was no evidence of dehydration, cardiac or renal insufficiency, hypothyroidism, adrenal insufficiency including cortisol and ACTH deficiency. The patient has also not received diuretic therapy. The patient was diagnosed with syndrome of inappropriate antidiuretic hormone (SIADH) and glimepiride was immediately discontinued." The patient was also not fluid restricted. His serum sodium promptly increased to $134 \mathrm{mmol} / \mathrm{L}$ over 12 days.

The authors point out that SIADH has been reported with first-generation sulfonylurea medications but not with second-generation sulfonylurea agents like glimepiride. The authors also conclude that while the patient's episode of SIADH is likely related to the administration of glimepiride, they cannot completely rule out the influence of other medications in the patient's regimen as contributing to the condition. They did measure hormones including $\mathrm{ADH}$, but they only obtained these labs once and so they cannot completely eliminate a possible subclinical hormone deficiency. The authors warn that clinicians need to consider hyponatremia in a patient receiving a second-generation sulfonylurea as possibly due to sulfonylurea-induced SIADH.

Adachi H, Yanai H. Adverse drug reaction. A possible case of glimepiride-induced syndrome of inappropriate antidiuretic hormone secretion [published online ahead of print January 2, 2015]. Diabetes Metab. http://dx.doi.org/10.1016/j.diabet.2014.11.010.

\section{MANIA ASSOCIATED WITH BORTEZOMIB ADMINISTRATION}

A 61-year-old female was diagnosed with multiple myeloma stage IIIB and was set to receive a chemotherapy regimen including bortezomib (Velcade). The patient was administered bortezomib $1.3 \mathrm{mg} / \mathrm{m}^{2}$ on days $1,8,15$, and 22 with dexamethasone $15 \mathrm{mg}$ intravenous (IV) infusion on the day and the day after bortezomib was administered, and thalidomide $75 \mathrm{mg}$ was given orally every night for 35 days. On the patient's ninth day of chemotherapy, she experienced a mental disorder. The patient was in high spirits, was irritable and restless, gave nonsensical answers to questions, and required less sleep; she also sometimes thought that she had great power to solve problems that she was not able to finish under normal circumstances.

Labs were drawn; the patient had a serum creatinine of $7.5 \mathrm{mg} / \mathrm{dL}$ (reference female value, $0.6-1.2 \mathrm{mg} / \mathrm{dL}$ ). She then received hemodialysis and continued with hemodialysis 3 times weekly. The patient's psychiatric symptoms improved after the first hemodialysis session, but this benefit was not seen with subsequent dialysis sessions. A CT of the patient's head was conducted and revealed a small infarction lesion that was not thought to cause the patient's psychiatric symptoms under normal conditions. Based on the findings, bortezomib was considered a possible cause of the patent's psychiatric symptoms.

The patient's dosage of bortezomib was reduced to $1 \mathrm{mg} / \mathrm{m}^{2}$ and her dexamethasone dosage was decreased to $10 \mathrm{mg}$, but the dosage of thalidomide was maintained. At this time, an MRI of the patient's head did not reveal the findings that would have been expected as myeloma cells infiltrate the brain. Therefore her mania was not thought to be due to a structural abnormality, but was drug induced.

After the dosage reduction and on the 16th day of chemotherapy, the patient experienced the same psychiatric symptoms that she had initially exhibited. 
Bortezomib and dexamethasone were discontinued and the patient was given a psychological evaluation. The psychiatrist diagnosed the patient with mania and started her on olanzapine $10 \mathrm{mg}$ daily and valproic acid $1 \mathrm{~g}$ daily for 30 days. The patient's mania symptoms were finally relieved, and she was started on an alternative chemotherapy regimen for her multiple myeloma. The patient received a new chemotherapy regimen of vincristine, doxorubicin, and dexamethasone. The patient did not have a recurrence of her mania with this new regimen.

The authors reviewed the possibility of psychiatric symptoms related to the patient's multiple myeloma. They state that the following mechanisms may be responsible for the patient's mania: direct infiltration of tumor cells in the brain, renal failure, hypercalcemia, and hypoxic ischemic encephalopathy. First, the results of the patient's CT and MRI do not support direct infiltration of tumor cells in the brain. Second, the patient's mania was relieved after her first hemodialysis session, but she relapsed soon after and additional hemodialysis sessions could not improve the symptoms. The authors ruled out renal failure as the etiology for the patient's mania. Third, hypercalcemia could possibly cause the patient's mania symptoms. The authors note that literature reports that when the serum calcium exceeds $18 \mathrm{mg} / \mathrm{dL}$ (reference range, $8.5-10.2 \mathrm{mg} / \mathrm{dL}$ ), it is possible to induce psychiatric symptoms. When this patient manifested mania symptoms, her serum calcium was in the range of 8.2 to $10.5 \mathrm{mg} \mathrm{dL}$. The authors also ruled out the possibility of hypercalcemia causing the patient's mania. Fourth, hypoxic ischemic encephalopathy could be a possible cause, but the patient's liver function was in the normal range and she had no jaundice or any typical behavior change related to hepatic encephalopathy. Therefore, the authors ruled out a possible cause of the patient's mania related to the liver.

The authors state that mania in a patient with multiple myeloma is relatively rare. They mention that the common adverse effects of bortezomib therapy are peripheral neuropathy, thrombocytopenia, neutropenia, and ECG abnormalities. The authors call for further preclinical studies focused on the mechanisms of multiple myeloma and bortezomib on the neuroendocrine system to direct rational drug use in multiple myeloma patients.

Jiang $\mathrm{H}, \mathrm{Hu} \mathrm{R}$, Miao M, et al. Mania in a patient with multiple myeloma after using bortezomib. J Med Cases. 2014;5(12):627-630. 